\title{
Requirements for Supporting Individual Human Creativity in the Design Domain
}

\author{
Uta Lösch $^{1}$, Julie Dugdale ${ }^{2}$ and Yves Demazeau ${ }^{3}$ \\ ${ }^{1}$ Institut AIFB, Universität Karlsruhe (TH), Germany, uta.loesch@aifb.uni-karlsruhe.de \\ ${ }^{2}$ LIG, Université Pierre Mendes France, Grenoble, France, Julie.Dugdale@imag.fr \\ ${ }^{3}$ LIG, CNRS, Grenoble, France, Yves.Demazeau@imag.fr
}

\begin{abstract}
Creativity is an important activity in many professional and leisure domains. This article presents a first step towards a system which will provide a set of tools for enhancing the individual creative abilities of the user in a design task. We have identified aspects which are characterise individual creativity: motivation, domain knowledge, externalization, inspiration and analogies, and requirements handling. Based on these aspects we have defined requirements and suggest associated system functionalities.
\end{abstract}

\section{Introduction}

In both our personal and professional lives creativity plays an important role. Some domains, such as art, are more closely associated with creativity than others. Nevertheless, finding creative solutions to problems is a common activity in many work situations. One domain that requires a creative approach is design, for example the design of physical objects, such as mobile devices, or less tangible objects such as web sites.

Previous work on using computers for supporting creativity has largely focused on either supporting group creativity ([4], [7], [14]) or has attempted to build systems that are inherently creative themselves ([10], [12], [15]). Unfortunately, there has been little work on supporting an individual's creativity for professional tasks. Misue and Tanaka [11] focus on the early stage of the creative process and have developed a pen and paper device that allows users to express their ideas and reconfigure sketches and concepts. Kules [9] examines how search engines can be used to support creativity. Pachet [13] proposes Interactive Musical Reflective Systems to support creativity. In these systems users explore their own musical style by teaching it to the system.

Despite these attempts tools for supporting individual creativity in the task of design are rare. The ultimate goal of our work is to develop a system to support individual human creativity. In this paper we define requirements for such a system. Section 2 describes the core aspects that characterise individual creativity. Section 3 addresses the specific task of design. Section 4 discusses the desired functionalities of a support tool. Section 5 concludes with a discussion. 


\section{Supporting Individual Creativity}

Creativity is an abstract notion that is hard to define. In this section we describe aspects that have been identified as being necessary preconditions for creativity.

\subsection{Motivation}

Motivation is a crucial aspect of creativity. It describes both the intrinsic and extrinsic reasons that lead to the engagement of the individual in a task. Intrinsic motivation occurs when an individual engages in an activity without an obvious external incentive, whereas extrinsic motivation is linked to some external incentive, such as money. Intrinsic motivation, in particular, has been shown to encourage creativity. It leads to curiosity and the readiness to take risks in deriving the solution [8]. Both of these factors increase the probability of creative results.

Intrinsic motivation is a precursor to the "flow experience" which is a mental state where a person is fully immersed in an activity and has a feeling of success in the process of that activity [3]. The flow experience is essential to the creative process and is characterised by four points: at each moment the goal of the individual engaged in the activity is clear; each action results in an immediate reaction; the difficulty of the task and the skills of the individual are balanced; focus on the task excluding all external distractions.

\subsection{Domain knowledge}

Knowledge of the domain is needed during the three main phases of the creative process: the preparation phase, the idea generation phase, and the validation phase. An understanding of the problem is constructed during the preparation phase where a sound knowledge of the domain and associated skills are crucial to creativity ([2], [16]). The idea generation phase is characterised by the occurrence of insights. An insight is the recognition of a new relationship or re-organization of knowledge and leads to new ideas regarding the problem solution. The occurrence of insights is impossible without domain knowledge [8]. The validation phase concerns judging the appropriateness of the creative solution in terms of its novelty and adaptability to a situation. This requires knowledge of what already exists in the domain and the intended environment [2].

\subsection{Externalisation}

Externalisation is the expression of thoughts or ideas in a form outside the physical boundary of the mind, e.g., by making notes or sketches. Externalisation is a way to extend the cognitive functions of the brain [8]. It helps to understand the problem and to produce new ideas by constructing or changing the mental representation of the problem. Externalisation also helps to organise and integrate information [11] and identify missing data. In the preparation phase externalisations 
are the first step towards a concrete representation of the problem [5]. In the idea generation phase, possible solutions are externalised in order to produce new insights or to elaborate ideas.

\section{Creativity Support in Design}

Design concerns defining the characteristics of an object or procedure so that it is adapted to a set of constraints [2]. Gero describes three types of design [6]. Routine designs are a small subset of the design solution space and are found by applying "good design practice" to define further constraints. Innovative designs are obtained if some variables in the design have unusual values. Creative designs lie outside the original search space. Boden's notions of p-creativity (psychological creativity) and h-creativity (historical creativity) can be translated into this model [1]. A result involves p-creativity if it is novel to the person who created the object, whereas hcreativity results in something that is novel to society. A p-creative design lies outside of one particular designer's design space whereas a h-creative design lies outside of all designers' design spaces. Finally, s-creativity (situated creativity) occurs when the result of a creative process contains unexpected ideas and the design lies outside of the initial design space of the problem [6].

\subsection{Inspiration and Analogies}

Obtaining inspiration and making analogies are valuables processes in creative design [2]. Sources of inspiration give us a better understanding of the problem and can introduce additional problem constraints. Presenting sources of inspiration appears to stimulate the search for analogies. Designers who have been confronted with possible analogies during the preparation phase tend to evoke more and a greater variety of sources than designers who have not been faced with possible sources of inspiration.

\subsection{Requirements Handling}

Requirements define the properties that a design solution must possess. Defining additional requirements reduces the space of possible design solutions and makes the solution more precise.

There are four kinds of requirements in the design domain [2]. Explicit requirements are directly derived from the problem description and are expressed in the specification. Constructed requirements are the expression of domain knowledge. Deduced requirements are obtained by analysing current requirements in order to deduce new ones. Induced requirements are those which are introduced by the designer from his or her understanding of the problem. The difference between constructed and induced requirements is that the former are not directly related to the specification but are the expression of the designer's knowledge, whereas induced requirements have their origin in the designer's interpretation of the specification. 
Some requirements are more important than others. Explicit requirements must be satisfied for each potential solution whereas preference requirements, such as colour may be less important.

\section{Requirements for Creativity Support}

This section suggests some functionality for a creativity support system based on the above factors.

\subsection{Functionalities to support motivation}

A system may motivate the users by addressing the four points concerning flow experience (section 2): The definition of goals and the users' awareness of them can be supported by structuring projects and defining sub-tasks. A basic way of providing immediate reactions to the users' actions is by using a WYSIWYG interface. However, more elaborate system reactions, such as having the system check if the user's input is coherent with previously defined design constraints, could be envisaged. To achieve a balance between the difficulty of the creative task and the skills of the user, users should be supported according to their level of expertise. To help users focus on their task, users should not have to deal with unrelated side activities and they should not be distracted. The system may support the first aspect by taking over routine tasks from the user, e.g. by providing templates of common designs. The second aspect is more difficult to support and could involve blocking the use of certain programs like e-mail. However, this is problematic since they may be used productively to support the design work.

Incentives or rewards provide a strong motivation for users. Different types of rewards already exist in certain systems, e.g. computer games. However, the same types of reward are not appropriate for professional systems. The issue of providing a suitable kind of reward remains an open question for us.

Finally, the system should be able to detect, resolve or prevent breakdowns when the user is stuck on a problem. This concerns the problems the user has with the creative aspect, rather than the use of the system. For example, a system could suggest appropriate design patterns to help the user overcome a breakdown.

\subsection{Functionalities for providing domain knowledge}

Domain knowledge is used in the three main phases of the creative process. In the problem understanding phase implicit requirements and constraints that are not included in the specification must be identified. These implicit requirements often concern design principles, such as accessibility. A creativity support tool should offer possibilities of finding information concerning the specific design domain, e.g. providing a domain manual or links to interesting sources.

The idea generation phase deals with the actual design. Here, design rules that exist in the domain have to be applied. Design rules are known solutions to frequently 
occurring problems. The support system should be able to detect situations where the application of design rules is possible and desirable.

In the evaluation phase the applicability of the solution is judged. This is judged in terms of the requirements that have been specified. A support tool could check whether the design conforms to the pre-specified requirements.

Another issue concerns the presentation of domain knowledge. Other modes besides text, such as images, animation or sound should be considered.

\subsection{Functionalities to support externalizations}

Two aspects that are important to externalizations are the expression of ideas anywhere and anytime and interaction protocols (i.e. the way users interact with the support tool).

Since ideas can occur unexpectedly a ubiquitious note-taking functionality is very useful. A related problem is how users later find their notes.

How the user interacts with the system will greatly influence the solutions created. The user should be able: to manipulate the design object as directly as possible; to interpret the representation created in the system in different ways (these reinterpretations will help the user to be creative); to be able to annotate the design [17].

The support system should record a history of events. This addresses the idea that design solutions are progressively developed. A designer may investigate different design solutions in parallel, or return to a previous partial solution and continue work.

\subsection{Functionalities related to providing inspiration and analogies}

Humans find it difficult to make analogies (i.e. transfer the properties from a source to a current solution) if the source of inspiration and the object to which the property is transferred have no common properties. A creativity support tool should allow users to access a set of solutions from the same and other domains in order to provide possible sources of inspiration and realize analogies. These solutions could be organized as a dynamic graphical library which may be searched based on an ontological description of their most relevant properties. The tool should also be able to identify interesting sources of inspiration based on the currently defined requirements and the current state of the solution.

\subsection{Functionalities related to requirements handling}

A design specification is used to derive an initial set of requirements. The creativity support tool could help in translating a specification to a set of requirements and with modifying the requirements during the design process. The support tool could also check whether the requirements are fulfilled by the proposed solution, detect any incoherencies in the set of requirements and make suggestions concerning their solution. Requirements may be interlinked, i.e. the modification of one requirement leads to the modification of other requirements. The system could be able 
to detect these links and to take them into account when searching for solutions to incoherencies. Since some requirements are more important than others the tool could label potential design solutions by their severity in breaking certain requirements.

\section{Conclusions and future work}

This article has discussed issues related to creativity and requirements for a tool to support individual human creativity in the domain of design. We currently develop a multi-agent system architecture that realizes the identified requirements. In the future, we will explore how the system may be personalized for different users.

\section{References}

1. Boden, M.: The Creative Mind: Myths and Mechanisms. Cardinal (1990)

2. Bonnardel, N.: Créativité et conception. Solal Editions (2006)

3. Csikszentmihalyi, M.: Creativity: Flow and the Psychology of Discovery and Invention. Harper Perennial (2006)

4. Farooq, U. et al.: Supporting creativity in distributed scientific communities. Proc. of the 2005 Int. Conf. on Supporting Group Work (GROUP'05, 217-226 (2005)

5. Fischer, G.: Symmetry of Ignorance, Social Creativity, and Meta-Design. Int. J. on Knowledge-Based Systems 13, 527-537 (2000)

6. Gero, J.: Computational Models of Creative Designing Based on Situated Cognition. Proc. of the $4^{\text {th }}$ Conf. on Creativity \& Cognition (C\&C2002), 3-10 (2002)

7. Hermann, T.: Design Issues for Supporting Collaborative Creativity. Proc. of the $8^{\text {th }}$ Int. Conf. on the Design of Cooperative Systems (2008)

8. Hewett, T et al.: Creativity Support Tool Evaluation Methods and Metrics. Proc. of the NSF Workshop on Creativity Support Tools, 10-24 (2005)

9. Kules, B.: Supporting Creativity with Search Tools. Proc. of the NSF Workshop on Creativity Support Tools, 53-64 (2005)

10. Lirong, Q. et al.: A multi-agent system supporting creativity in conceptual design. Proc. Of the $8^{\text {th }}$ Int. Conf. on Computer-Supported Cooperative Work in Design (2003)

11. Misue, K. and Tanaka, J.: A Handwriting Tool to Support Creative Activities. Proc. of the $9^{\text {th }}$ Int. Conf. on Knowledge-Based Intelligent Information and Engineering Systems (KES2005), 423-429 (2005).

12. Oliveira, H. et al.: Tra-la-lyrics: An Approach to Generate Text Based On Rhythm. Proc. Of the $4^{\text {th }}$ Int. Joint Workshop on Computational Creativity (2007)

13. Pachet, F.: Enhancing Individual Creativity with Interactive Musical Reflective Systems. Musical Creativity: Current Research in Theory and Practice (2004)

14. Pinho, D. et al.: Similarity-based agents for design. Proc. of the $9^{\text {th }}$ Int. Conf. on ComputerSupported Cooperative Work in Design, page 417-422 (2006)

15. Strapparava, C. et al.: Automatizing two creative functions for advertising. Proc. of the $4^{\text {th }}$ Int. Joint Workshop on Computational Creativity (2007)

16. Warr, A. and O'Neill, E.: Understanding Design as a Social Creative Process. Proc. of the $5^{\text {th }}$ Conf. on Creativitvy \& Cognition (C\&C'05), page 118-127 (2005)

17. Yamamoto, Y. and Nakakoji, K.: Interaction Design of Tools for Fostering Creativity in the Early Stages of Information Design. Int. J. of Human-Computer Studies (IJHCS), 63 (4-5), $515-535(2005)$ 